\title{
Spin in a Closed-Shell Organic Molecule on a Metal Substrate Generated by a Sigmatropic Reaction
}

\author{
Marie-Laure Bocquet,* Nicolas Lorente, Richard Berndt, and Manuel Gruber
}

\begin{abstract}
Inert metal surfaces present more chances of hosting organic intact radicals than other substrates, but large amounts of delocalized electronic states favor charge transfer and thus spin quenching. Lowering the molecule-substrate interaction is a usual strategy to stabilize radicals on surfaces. In some works, thin insulating layers were introduced to provide a controllable degree of electronic decoupling. Recently, retinoid molecules adsorbed on gold have been manipulated with a scanning tunneling microscope (STM) to exhibit a localized spin, but calculations failed to find a radical derivative of the molecule on the surface. Now the formation of a neutral radical spatially localized in a tilted and lifted cyclic end of the molecule is presented. An allene moiety provokes a perpendicular tilt of the cyclic end relative to the rest of the conjugated chain, thus localizing the spin of the dehydrogenated allene in its lifted subpart. DFT calculations and STM manipulations give support to the proposed mechanism.
\end{abstract}

It to design sustainable pure organic magnets to replace metals. Potential candidates are spin $1 / 2$ molecula radicals. $^{[1,2]}$ Despite recent advances ${ }^{[2,3]}$ in depositing multilayers of these species, the open-shell structure of radicals renders them difficult to work with. To date a few classical radicals, such as nitric oxide, ${ }^{[4,5]}$ 1,3,5-triphenyl-6-oxoverdazyl (TOV), ${ }^{[6]}$ polychloro triphenyl methyl, ${ }^{[3,7-9]}$ and bis-diphenylene-phenyl allyl (BDPA) ${ }^{[10]}$ radicals have been reported to be stable on metal surfaces. Typically, intact radicals can be deposited on metals that show low reactivity, such as the relatively inert $\mathrm{Au}(111)$ surface. ${ }^{[11,12]}$ Besides depositing the radicals, detecting them requires spin-polarized spectroscopy

[*] Dr. M.-L. Bocquet

PASTEUR, Département de chimie, École normale supérieure

PSL University, Sorbonne Université, CNRS

75005 Paris (France)

E-mail: marie-laure.bocquet@ens.fr

Prof. Dr. N. Lorente

Centro de FIsica de Materiales CFM/MPC (CSIC-UPV/EHU)

Paseo Manuel de Lardizabal 5

20018 Donostia-San Sebastian (Spain)

and

Donostia International Physics Center (DIPC)

Paseo Manuel de Lardizabal 4

20018 Donostia-San Sebastian (Spain)

Prof. Dr. R. Berndt, Dr. M. Gruber

Institut für Experimentelle und Angewandte Physik

Christian-Albrechts-Universität

24098 Kiel (Germany)

(2) Supporting information and the ORCID identification number(s) for the author(s) of this article can be found under:

https://doi.org/10.1002/anie.201812121. techniques. Photon-emission techniques are well adapted for the study of overlayers of radical molecules. ${ }^{[2]}$ Furthermore, creating local spins with eventual patterning for application is of great interest for the study of the inorganic-organic spinterface. ${ }^{[13,14]}$ To this end, scanning probes can locally produce molecular radicals.

Recently, the closed-shell molecule retinoic acid ( $\mathrm{ReA}$, see Scheme 1) was deposited onto a crystalline gold(111) surface and then subjected to current pulses at negative sample voltage of approximately $2.5 \mathrm{~V} .^{[15,16]}$ This procedure reproducibly led to intriguing changes of the molecule. The apparent height of the cyclic end of ReA (denoted "head" below) increased by $140 \mathrm{pm}$ and the head group appeared laterally shifted while the rest of the molecule (denoted "tail") remained virtually unaltered. Surprisingly, this contrast modification of the head part also led to a spatially localized Kondo feature in tunneling spectra. As expected for a localized spin, this feature could be tuned by an external magnetic field. These results suggest that it may be possible to bottom-up assemble arrays of switchable spins on surfaces.

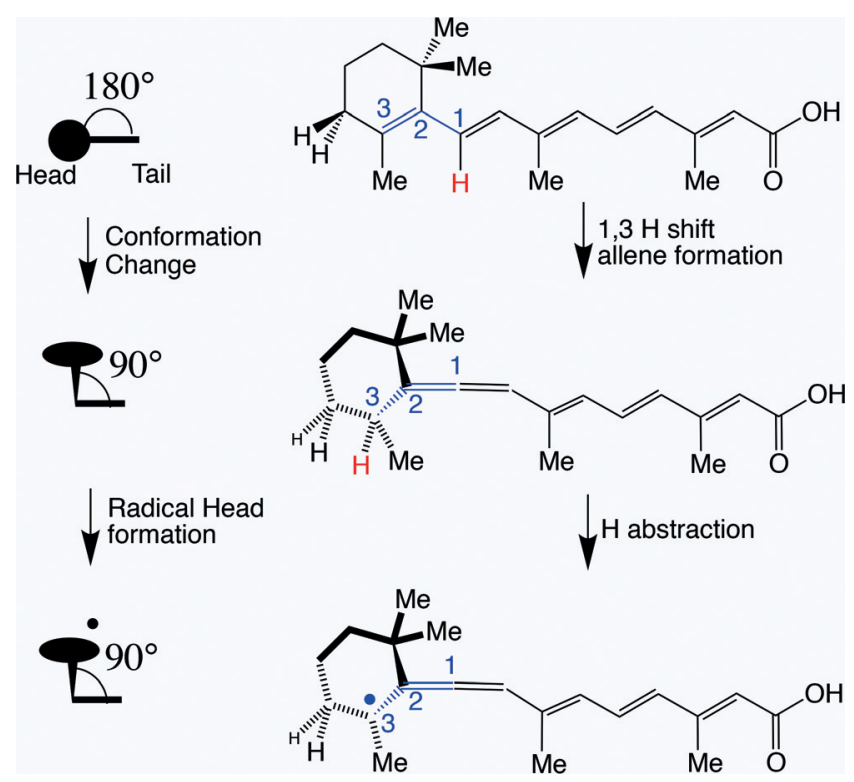

Scheme 1. Two-step chemical reaction of retinoic acid ReA (top) leading to the magnetic product molecule ALRAD (bottom). The reactant $\mathrm{ReA}$ consists of a head (substituted cyclohexene) and a tail (linear conjugated chain). Left: sketches of the topological changes of ReA, modelled by a ball (head) and a stick (tail), to the neutral intermediate $A L$ (middle) and to the radical ALRAD, for which the ball and the stick are perpendicular to each other. Right: corresponding mechanisms where the labile (moving and removed) hydrogen atom is marked in red. Three carbon atoms labeled in blue are discussed in the text. 
The DFT calculations ${ }^{[15]}$ were unable to find a radical on the surface that could explain the experimental data. Herein, we provide for the first time an explanation of the requirements and mechanisms leading to a stable radical after STM manipulation.

We present a reaction pathway (not reported on surface chemistry) that leads to spin localization in ReA on a metal substrate as observed in the STM experiments. The final reaction product is a neutral radical. It is formed by loss of a hydrogen atom from an allene intermediate that is produced by a concerted hydrogen sigmatropic rearrangement of the ReA molecule. The proposed mechanism is corroborated by DFT calculations and STM contact measurements allowing reversible switching. Our findings show how organic radicals may be stabilized on metallic surfaces without resorting to insulating layers, which tend to weakly bind organic species.

The high current densities and electrical field strength present in a STM junction have been widely used to induce chemical processes on surfaces with molecular precision. In particular, intramolecular hydrogen transfers and dehydrogenations have been already reported from $\mathrm{N}-\mathrm{H}$ or $\mathrm{C}-\mathrm{H}$ bonds of porphyrinic molecules, ${ }^{[17-24]}$ melamine, ${ }^{[25]}$ and nanographene-like molecules. ${ }^{[26,27]}$ The proposed two-step process depicted in Scheme 1 (right) is motivated by the general concept of hydrogen lability in conjugated molecules but selects a pathway associated to a major topological change (left). First in a sigmatropic reaction, a hydrogen atom (red in Scheme 1) migrates along an allyl fragment between $\mathrm{C} 1$ and $\mathrm{C} 3$ of the endocyclic bond connecting $\mathrm{C} 1$ and $\mathrm{C} 2 .{ }^{[28]}$ Interestingly the migrating hydrogen atom resides in an allylic position with respect to the constrained endocyclic double bond. As a result of the cyclic constraint, the endocyclic double bond is lifted away from the linear conjugated chain putting the allylic hydrogen in the chain close to a favorable anti configuration as required in such sigmatropic reactions. After the [1,3] hydrogen shift, an allene or a cumulative diene is formed imposing a drastic conformational tilt of $90^{\circ}$ on the cyclic end. In a second step, a radical is produced at the cyclic end by abstraction of the labile hydrogen atom (red in Scheme 1). This two-step process involves a $90^{\circ}$ rotation of the cyclic head part and also disrupts the $\pi$ system between the head and the tail imposed by the allene structure. Such an allenic rearrangement has previously been suggested for retinoid molecules. ${ }^{[29]}$

Notably the reaction involves four $\pi$ electrons (one electron of the labile $\mathrm{H}$ atom and one electron per carbon atom of the allyl $\mathrm{C} 1$ to $\mathrm{C} 3$ fragment) and is therefore not thermally activated according to the Woodward-Hoffman rules. It rather requires an electronic excitation to provoke the hydrogen migration and the current pulses in the STM experiments serve this purpose.

We used ab initio calculations to test and validate our chemical hypothesis in terms of adsorption structures and the stability, magnitude and spatial distribution of the resulting magnetism (see the Methods Section and Supporting Information, Figure S1).

The intermediate AL and the product ALRAD adsorbed on $\mathrm{Au}(111)$ have been investigated within the DFT framework. Their optimized geometries and associated enthalpy barriers are shown in Figure 1. Both steps are endothermic. The sigmatropic reaction of one hydrogen is less costly $(0.84 \mathrm{eV})$ than the dissociation of one $\mathrm{C}-\mathrm{H}$ bond $(1.29 \mathrm{eV})$, which is lower than the gas-phase dissociation energy because the dissociated $\mathrm{H}$ is adsorbed to the surface, reducing the dissociation barrier. These thermodynamic barriers are compatible with the excitation energy of $2.5 \mathrm{eV}$ provided by the current/voltage pulse. The adsorbed ALRAD possess 0.55 Bohr magneton (less than one unpaired electron) showing a partial quenching of magnetization upon adsorption. This is clearly illustrated in the Supporting Information, Figure S1 by the projected density of states on the spinpolarized carbon atoms whose integration until the Fermi energy is the partial occupation. This is a way to estimate the molecular interaction with the substrate. The residual magnetism is spatially localized in the perpendicular $\pi$ system resembling the SOMO of a $\mathrm{C} 1$-to-C3 conjugated radical at the cyclic side of the allene molecule. This is shown in the spin density in Figure 1e. Clearly, the spin density is lifted away from the surface explaining the spin partial preservation in proximity to the metallic surface.

How compatible is this scenario with the STM-data? The first step involves the migration of an allylic hydrogen atom with respect to the endocyclic double bond and requires electronic excitation. Remarkably there are two more allylic hydrogen atoms in the cycle (belonging to the $\mathrm{CH}_{2}$ ) that could migrate and lead to additional states of the molecule. Indeed, several switched states were observed in the experiments. ${ }^{[15]}$ In these cases no allene is formed and no large conformational change should occur, which again is in qualitative agreement with the experimental observations. Furthermore,

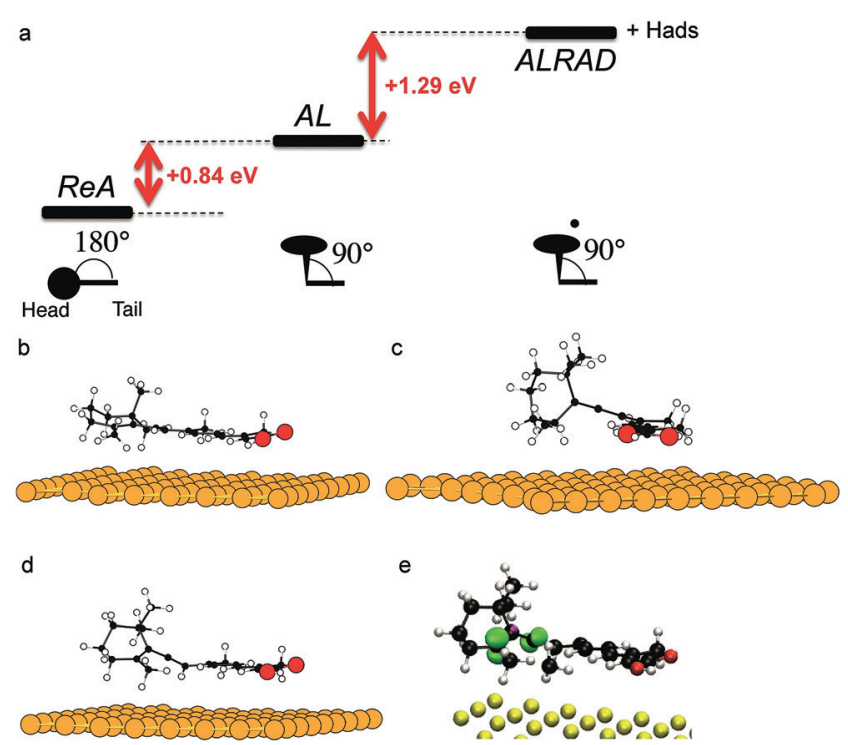

Figure 1. a) Enthalpy profile in eV of the two-step mechanism of $\operatorname{ReA}$ on $A u(111)$ calculated with DFT. The first step is the formation of the allene (AL) with an enthalpy $0.84 \mathrm{eV}$ above the reactant. In the second step, the migrating hydrogen atom is detached and adsorbed to the substrate (Hads) costing only $1.29 \mathrm{eV}$. This leaves behind a radical species (ALRAD) at a total enthalpy cost of $2.13 \mathrm{eV}$. b)-d) Snapshots of the DFT optimized structures of ReA, AL, and ALRAD respectively. e) Side view of the spin density map of ALRAD. The spin density of $0.05 \mathrm{eV} \AA^{-3}$ is represented by a light green contour. 
we cannot exclude a similar migration of one methyl substituent in allylic and axial positions with respect to the endocyclic double bond. Hence, it is impossible to unambiguously identify all final products that may be experimentally accessible. However, it is clear that the invoked sigmatropic reactions are versatile enough to account for the observed diversity of switched molecular states.

As described above, allene formation involves a rotation of the cyclic head part, which increases the height of this part of the molecule. This matches the experimental observations (Figure 2) ${ }^{[15]}$ that the apparent height of molecules in the Kondo state is much higher than in their pristine state.

At first glance, a mechanism based on $\mathrm{H}$ abstraction seems to be inconsistent with the reported reversibility of the switching of ReA. In the experiments, the transition from pristine ReA to a state displaying a Kondo effect (step 1) is easily achieved by applying suitable voltage/current pulses. Returning to a state without Kondo signature (step 2) is possible but more complicated. This is illustrated in Figure 2. Attempts to switch ReA molecules back to their pristine state via voltage pulses are usually inefficient as shown in Figures $2 \mathrm{a}-\mathrm{c}$. Upon a voltage pulse of a few seconds (ca. $-1 \mathrm{~V} ; 4 \mathrm{nA})$ on the molecule indicated by the arrow, the image of the molecule is changed and resembles that of pristine reference molecules. However, the differential conductance spectrum of the switched molecule (indicated by an arrow in Figure 2b) still exhibits a Kondo resonance (blue

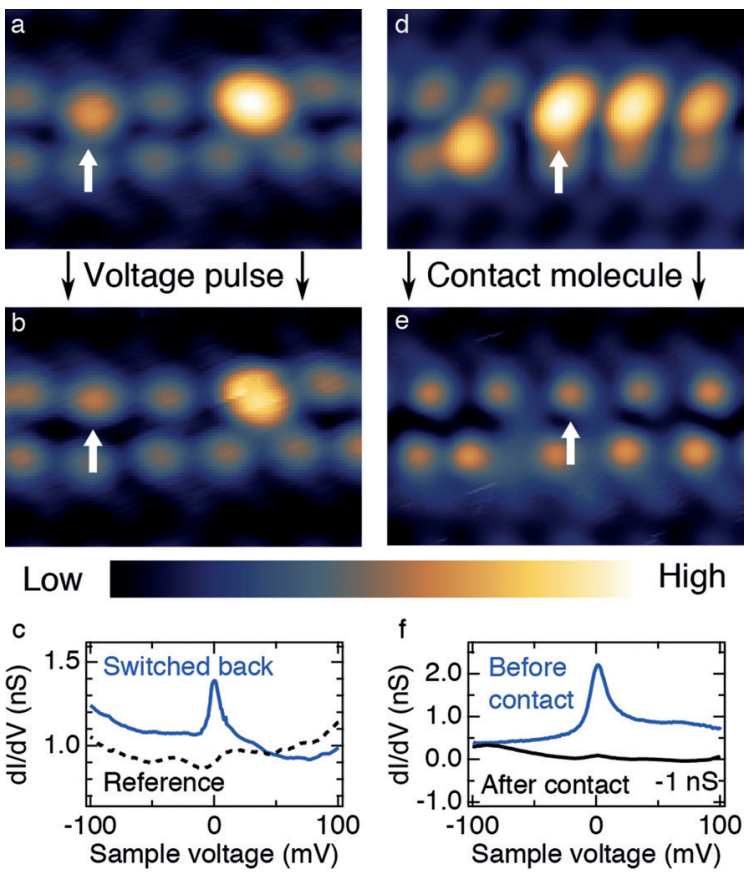

Figure 2. STM topographs of double rows of ReA molecules on Au(111) a) before and b) after a reverse-switching attempt via voltage pulse (ca. $-1 \mathrm{~V} ; 4 \mathrm{nA}$ ). Note both (a) and (b) display pristine and switched ReA molecules. c) Differential-conductance spectra of the switched molecule (blue line, molecule indicated by an arrow in (b) and of a pristine reference molecule (dashed black). STM topographs d) before and e) after gently contacting molecules with the STM tip. f) $d I / d V$ spectra of the molecule indicated by an arrow in (d) and (e) before (blue) and after (black) contact. The black curve is vertically shifted by $1 \mathrm{nS}$ for clarity. curve in Figure 2c). In other words, the attempt to induce back-switching did result in a change of conformation but did not significantly modify the electronic structure of the molecule near the Fermi level. Closely related observations were often made. The change of the STM contrast of the molecules is consistent with 1) either a change of adsorption geometry of the molecule or a different arrangement of the allylic hydrogens within the molecule and 2) the absence of $\mathrm{H}$ transfer to the molecule, the latter being required to return to the pristine non-magnetic state.

Actually, $\mathrm{H}$ transfer to the molecule is most probably limiting the efficiency of the back-switching. While $\mathrm{H}_{2}$ is the most abundant residual gas in ultra-high vacuum systems and therefore present on surfaces at $5 \mathrm{~K}^{[30,31]}$ the hydrogen molecule needs to be dissociated and transferred to the adsorbed ReA during back-switching. This may be the reason underlying the low-success rate of the reverse switch via voltage pulses. Nonetheless, there is clear indication of $\mathrm{H}$ being present at the STM tip, which allows, for instance, $\mathrm{H}$ transfer from the tip to single $\mathrm{Fe}$ atoms on $\mathrm{Au}(111)$ during contact formation. ${ }^{[32]}$ In the present case, we used a similar approach, that is, we contacted the molecules to transfer $\mathrm{H}$ more efficiently. The process is illustrated in Figures $2 \mathrm{~d}-\mathrm{f}$. Four molecules were successively gently contacted with the STM tip. The subsequent topograph (Figure 2e) shows that images of the initially switched molecules have been converted to the typical shape and height of the pristine molecule. In addition, this method of reverse-switching is considered as complete, as the contacted molecules do no longer exhibit a Kondo resonance (Figure 2f).

The produced radicals are stable on the surface over hours (Supporting Information, Figure S2). Only after contacting the radical species was a conversion to the original nonradical state observed. We emphasize here the need of an inert substrate such as $\mathrm{Au}(111)$. On more reactive surfaces such as on $\mathrm{Ag}$ and $\mathrm{Cu}$ (Supporting Information) we could not find radicals after manipulation with the STM tip.

In conclusion, DFT calculations show that ReA on a $\mathrm{Au}$ surface may be converted into a long-lived organic radical by an STM-induced reaction. When the molecule is activated through suitable voltage/current pulses, allylic hydrogen from the endocyclic bond is likely to migrate and eventually dissociate, leading to a stable radical with the spin entirely localized to the cyclohexene group. The proposed mechanism is consistent with all experimental observations. In recent STM manipulation experiments, cholesterol, a molecule with a cyclohexene group embedded into several cyclohexane groups, has likewise been switched into a state displaying a Kondo effect. ${ }^{[33]}$ On some other molecules with a cyclohexene unit like shikimic acid ${ }^{[34]}$ related attempts remained without success. We speculate that a keto-enol tautomerism involving the endocyclic bond may be a competing reaction path in the unsuccessful cases. A search for further molecules that may be switched on surfaces to a state with a localized spin is in progress. The use of local probes to induce reactions on organic layers allows us to achieve an enhanced control of charges and spins at the interface between a molecular layer and a solid substrate. The conversion of a single molecule into a stable radical in an otherwise closed-shell molecular layer, 
provides new possibilities to create hierarchical structures in a spinterface that combine radicals and closed shell molecules.

\section{Methods}

DFT calculations were performed for a periodic slab geometry as implemented in the VASP code. ${ }^{[35]}$ Exchange and correlation were treated with the PBE form of the generalized gradient approximation. ${ }^{[36]}$ Dispersion interactions were modeled with the method by Tkatchenko and Scheffler. ${ }^{[37]}$ The atomic positions of the two topmost Au layers from the six-layer Au slab and the molecules in the $(7 \times 3)$ $\mathrm{Au}(111)$ supercell $(17.26 \times 9.97 \times 25.85 \AA)$ were relaxed until forces were smaller than $50 \mathrm{meV}^{-1}$.

The STM measurements were performed in a commercial (Createc) STM operated in ultra-high vacuum at $5 \mathrm{~K}$. All STM topographs were acquired in constant-current mode. The $\mathrm{d} I / \mathrm{d} V$ spectra were acquired with a lock-in amplifier using a voltage modulation of $2.8 \mathrm{mV}$ amplitude.

\section{Acknowledgements}

We thank Sven Johannsen for auxiliary measurements. M.L.B. thanks the national computational center CINES, and TGCC (Grant 2017- [GENCI project: A0010807364]) for a computer grant and acknowledges the Mercator fellowship of SFB 677 in CAU Kiel. N.L. acknowledges financial support from MINECO (MAT2015-66888-C3-2-R) and FEDER funds, and he is thankful to the Mercator fellowship of SFB 677 in CAU Kiel. M.G. acknowledges financial support of SFB 677. This project has received funding from the European Union's Horizon 2020 research and innovation program under grant agreement No. 766726.

\section{Conflict of interest}

The authors declare no conflict of interest.

Keywords: density functional calculations - metal surfaces . organic radicals · scanning tunneling microscopy · sigmatropy

[1] M. Mas-Torrent, N. Crivillers, C. Rovira, J. Veciana, Chem. Rev. 2012, 112, 2506-2527.

[2] M. B. Casu, Acc. Chem. Res. 2018, 51, 753-760.

[3] V. Mugnaini, A. Calzolari, R. Ovsyannikov, A. Vollmer, M. Gonidec, I. Alcon, J. Veciana, M. Pedio, J. Phys. Chem. Lett. 2015, 6, 2101-2106.

[4] R. Requist, S. Modesti, P. P. Baruselli, A. Smogunov, M. Fabrizio, E. Tosatti, Proc. Natl. Acad. Sci. USA 2014, 111, 69-74.

[5] Y.-h. Zhang, S. Kahle, T. Herden, C. Stroh, M. Mayor, U. Schlickum, M. Ternes, P. Wahl, K. Kern, Nat. Commun. 2013, 4, 2110

[6] J. Liu, H. Isshiki, K. Katoh, T. Morita, B. K. Breedlove, M. Yamashita, T. Komeda, J. Am. Chem. Soc. 2013, 135, 651-658.

[7] C. Simão, M. Mas-Torrent, N. Crivillers, V. Lloveras, J. M. Artés, P. Gorostiza, J. Veciana, C. Rovira, Nat. Chem. 2011, 3, 359.
[8] R. Frisenda, R. Gaudenzi, C. Franco, M. Mas-Torrent, C. Rovira, J. Veciana, I. Alcon, S. T. Bromley, E. Burzurí, H. S. J. van der Zant, Nano Lett. 2015, 15, 3109-3114.

[9] L. Yuan, C. Franco, N. Crivillers, M. Mas-Torrent, L. Cao, C. S. S. Sangeeth, C. Rovira, J. Veciana, C. A. Nijhuis, Nat. Commun. 2016, 7, 12066.

[10] S. Müllegger, M. Rashidi, M. Fattinger, R. Koch, J. Phys. Chem. C 2013, 117, 5718-5721.

[11] S.-A. Savu, I. Biswas, L. Sorace, M. Mannini, D. Rovai, A. Caneschi, T. Chassé, M. B. Casu, Chem. Eur. J. 2013, 19, $3445-$ 3450.

[12] F. Grillo, V. Mugnaini, M. Oliveros, S. M. Francis, D.-J. Choi, M. V. Rastei, L. Limot, C. Cepek, M. Pedio, S. T. Bromley, N. V. Richardson, J.-P. Bucher, J. Veciana, J. Phys. Chem. Lett. 2012, 3 , $1559-1564$.

[13] S. Sanvito, Nat. Phys. 2010, 6, 562.

[14] M. Cinchetti, V. A. Dediu, L. E. Hueso, Nat. Mater. 2017, 16, 507.

[15] S. Karan, N. Li, Y. Zhang, Y. He, I. P. Hong, H. Song, J.-T. Lü, Y. Wang, L. Peng, K. Wu, G. S. Michelitsch, R. J. Maurer, K. Diller, K. Reuter, A. Weismann, R. Berndt, Phys. Rev. Lett. 2016, 116, 027201 .

[16] M. Gruber, A. Weismann, R. Berndt, J. Phys. : Condens. Matter 2018, 30, 424001 .

[17] A. Zhao, Q. Li, L. Chen, H. Xiang, W. Wang, S. Pan, B. Wang, X. Xiao, J. Yang, J. G. Hou, Q. Zhu, Science 2005, 309, 1542-1544.

[18] P. Liljeroth, J. Repp, G. Meyer, Science 2007, 317, 1203-1206.

[19] A. Sperl, J. Kröger, R. Berndt, Angew. Chem. Int. Ed. 2011, 50, 5294-5297; Angew. Chem. 2011, 123, 5406-5409.

[20] W. Auwärter, K. Seufert, F. Bischoff, D. Ecija, S. Vijayaraghavan, S. Joshi, F. Klappenberger, N. Samudrala, J. V. Barth, Nat. Nanotechnol. 2011, 7, 41.

[21] a) T. Kumagai, F. Hanke, S. Gawinkowski, J. Sharp, K. Kotsis, J. Waluk, M. Persson, L. Grill, Nat. Chem. 2013, 6, 41; b) H. Böckmann, S. Gawinkowski, J. Waluk, M. B. Raschke, M. Wolf, T. Kumagai, Nano Lett. 2018, 18, 152-157.

[22] Y. F. Wang, J. Kröger, R. Berndt, H. Vázquez, M. Brandbyge, M. Paulsson, Phys. Rev. Lett. 2010, 104, 176802.

[23] J. Kügel, A. Sixta, M. Böhme, A. Krönlein, M. Bode, ACS Nano 2016, 10, 11058-11065.

[24] J. Kügel, L. Klein, M. Leisegang, M. Bode, J. Phys. Chem. C 2017, 121, 28204-28210.

[25] S. Pan, Q. Fu, T. Huang, A. Zhao, B. Wang, Y. Luo, J. Yang, J. Hou, Proc. Natl. Acad. Sci. USA 2009, 106, 15259-15263.

[26] H. Lesnard, M.-L. Bocquet, N. Lorente, J. Am. Chem. Soc. 2007, $129,4298-4305$.

[27] M. Treier, C. A. Pignedoli, T. Laino, R. Rieger, K. Müllen, D. Passerone, R. Fasel, Nat. Chem. 2010, 3, 61.

[28] R. B. Woodward, R. Hoffmann, Angew. Chem. Int. Ed. Engl. 1969, 8, 781-853; Angew. Chem. 1969, 81, 797-869.

[29] J. Sueiras, W. H. Okamura, J. Am. Chem. Soc. 1980, 102, $6255-$ 6259.

[30] F. D. Natterer, F. Patthey, H. Brune, Surf. Sci. 2013, 615, 80-87.

[31] S. J. Altenburg, R. Berndt, New J. Phys. 2014, 16, 093047.

[32] M. Mohr, A. Weismann, R. Berndt, unpublished results.

[33] S. Karan, R. Berndt, Phys. Chem. Chem. Phys. 2016, 18, $9334-$ 9337.

[34] M. Gruber, R. Berndt, J. Phys. Chem. C 2016, 120, 18642-18650.

[35] G. Kresse, J. Furthmüller, Comput. Mater. Sci. 1996, 6, 15-50.

[36] J. P. Perdew, K. Burke, M. Ernzerhof, Phys. Rev. Lett. 1996, 77, $3865-3868$.

[37] A. Tkatchenko, M. Scheffler, Phys. Rev. Lett. 2009, 102, 073005. 